\title{
Self-Harm in Children and Adolescents: An Update on Key Issues
}

Keith Hawton

Prof. Keith Hawton is a Director of the Centre for Suicide Research at the University of Oxford. For more than 40 years he and his research group have been conducting investigations concerning the causes, treatment, prevention and outcome of suicidal behaviour. Prof. Hawton has received research awards from all the major international suicide prevention organisations. His interests include epidemiology and clinical management of self-harm, suicide and self-harm in adolescents, media influences on self-harm and evaluation of suicide prevention initiatives. Key factors that motivate him to continue working in the field of suicidology are the emergence of new challenges and phenomena, such as the role of the internet in suicidal behaviour, new types of suicidal behaviour; the satisfaction of making contributions to prevention of suicide; rewarding collaborations with a wide range of people, both nationally and internationally; and the fact that the field attracts many people who share the same values.

Abstract. Evidence from several countries highlights increasing rates of both self-harm and suicide in young people. In England for example, rates of self-harm have recently increased very substantially in female adolescents, especially those aged under 17 years of age. Also rates of suicide have increased in under-20 year-olds of both genders over the past decade. As in adults, self-harm is an important risk factor for future suicide. There is a pressing need therefore to identify contributory factors that may assist in the design of prevention and treatment initiatives to try to reduce this phenomenon and to help ameliorate its consequences for young people. This has become all the more pressing with the many difficulties presented by the Covid-19 pandemic. In this presentation the epidemiology of self-harm and suicide will be discussed in the context of the iceberg model. Some key factors that contribute to self-harm will then be considered. These include evidence about the important role of puberty in the onset of self-harm, and the contribution of psychiatric disorders, mood instability and sleep disorders. Self-harm and suicide in children and adolescents often appears to spread between individuals, with clusters of the behaviour being far more common than in adults. While media influences on self-harm and suicide continue to be important in young people, the internet and social networking are making an increasing 
contribution, with some positive aspects, as well as negative ones. Recent findings on the association between self-harm and subsequent suicide will be presented, highlighting the long-term risk of suicide in children and adolescents and the fact that there is often a change of method between acts of self-harm and eventual suicide. The impact of self-harm on families will be considered. The presentation will conclude with a summary of key challenges for prevention, including a discussion of the issue posed by the Covid-19 pandemic. 